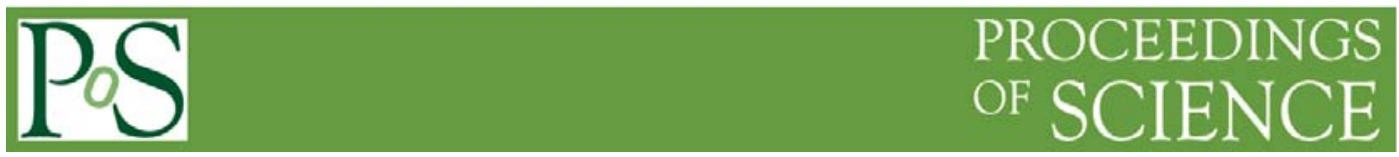

\title{
Precision Timing with the CMS MIP Timing Detector
}

\section{Adi Bornheim ${ }^{1}$}

Caltech

1200 E California Blvd, Pasadena, California, USA

E-mail: bornheim@caltech.edu

The Compact Muon Solenoid (CMS) detector at the CERN Large Hadron Collider (LHC) is undergoing an extensive Phase II upgrade program to prepare for the challenging conditions of the High-Luminosity LHC (HL-LHC). In particular, a new timing layer with hermetic coverage up to a pseudo-rapidity of $|\eta|=3$ will measure minimum ionizing particles (MIPs) with a time resolution of $\sim 30 \mathrm{ps}$. This MIP Timing Detector (MTD) will consist of a central barrel region based on LYSO:Ce crystals read out with SiPMs and two end-caps instrumented with radiationtolerant Low Gain Avalanche Detectors. The precision time information from the MTD will reduce the effects of the high levels of pile-up expected at the HL-LHC and will bring new and unique capabilities to the CMS detector. The time information assigned to each track will enable the use of 4D reconstruction algorithms and will further discriminate interaction vertices within the same bunch crossing to recover the track purity of vertices in current LHC conditions. For instance, in the analysis of di-Higgs boson production decaying to two b quarks and two photons, 30 ps timing resolution is expected to improve the effective luminosity by $22 \%$ through gains in b-tagging and photon isolation efficiency. We present motivations for precision timing at the HL-LHC and the ongoing MTD R\&D targeting enhanced timing performance and radiation tolerance.

European Physical Society Conference on High Energy Physics - EPS-HEP2019 -

10-17 July, 2019

Ghent, Belgium

\footnotetext{
${ }^{1}$ Speaker

(c) Copyright owned by the author(s) under the terms of the Creative Commons Attribution-NonCommercial-NoDerivatives 4.0 International License (CC BY-NC-ND 4.0).
} 


\section{Introduction}

The High-Luminosity LHC (HL-LHC) will deliver collisions with instantaneous luminosity of $5.0 \times 10^{34} \mathrm{~cm}^{-2} \mathrm{~s}^{-1}$ in the initial phase, and up to $7.5 \cdot 10^{34} \mathrm{~cm}^{-2} \mathrm{~s}^{-1}$ later on. This corresponds to an average number of simultaneous pp collisions per bunch crossing (pile up) of 140 and 200, respectively [1].

The challenge for Compact Muon Solenoid experiment (CMS) [2], one of two multi-purpose detectors to be operated on the LH-LHC, will be to keep the same level of performance as today, in terms of particle reconstruction and identification, in such a high pile up environment. The CMS global event description relies on efficient track-vertex and track-cluster assignment. It has been found that in events with a vertex linear density (computed along the beam axis) above $1 \mathrm{~mm}^{-1}$ the event reconstruction is severely degraded.

In order to mitigate the negative effect of HL-LHC pile up, CMS will add a novel timing detector, placed between the silicon tracker and the calorimeters, with a target resolution of 30 to 40 ps. This detector is optimized to measure the time of arrival of Minimum Ionizing Particles (MIP). This will allow to measure, in addition to the spatial position of each vertex, also its timestamp. The collisions in the luminous region of HL-LHC have a time spread of about 180 ps. The MTD will allow identifying charged particles which are incompatible with the time of the primary vertex and utilize this information in the event reconstruction. In Figure 1 we show an illustration of the implementation of the MTD in CMS and the expected track lines density at HL-LHC.

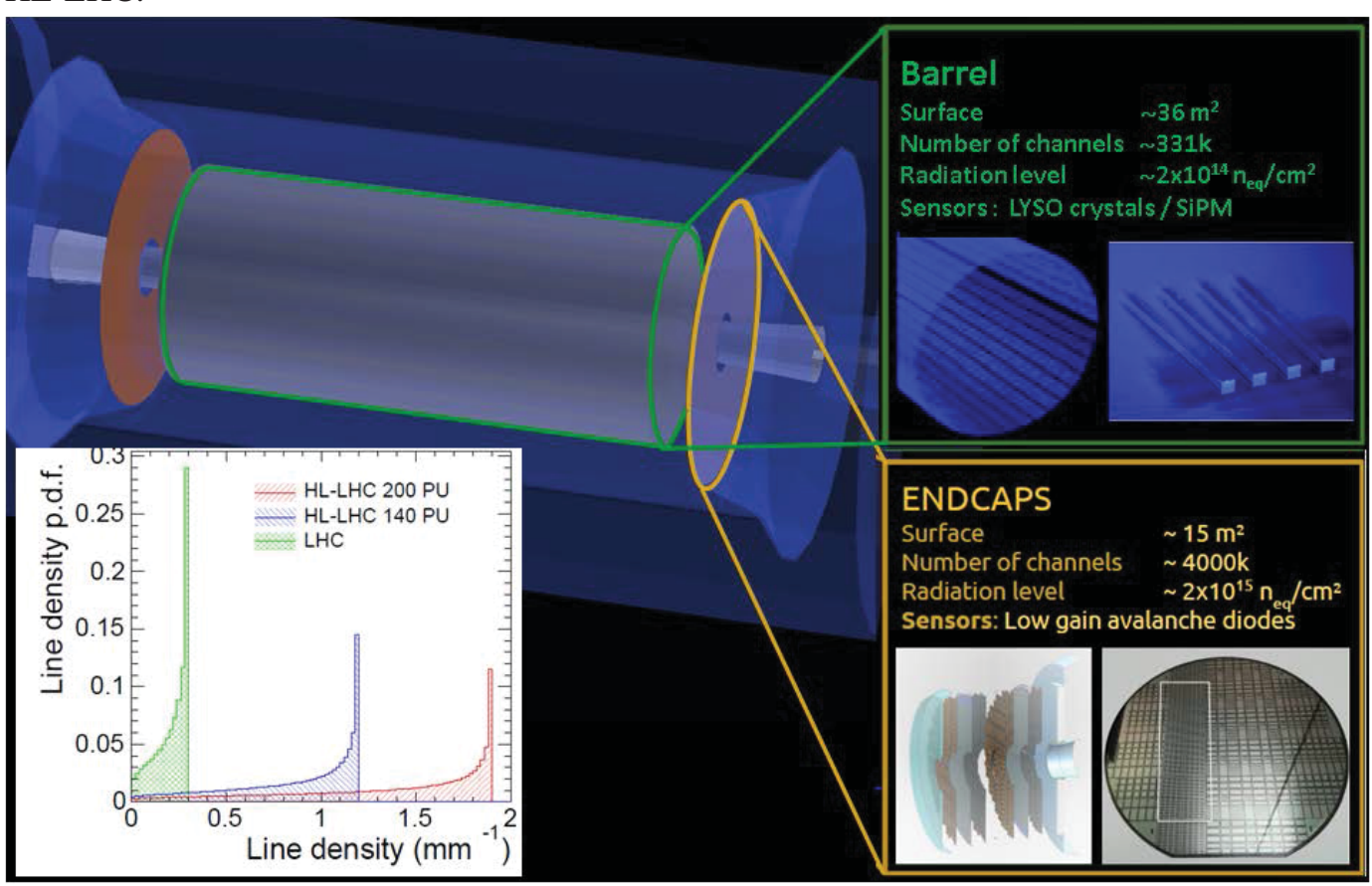

Figure 1 : Illustration of the implementation of the MTD detector inside CMS and the different technologies utilized in the barrel and the endcap section (insert) Probability density functions of the line density along the beam axis for the pileup of about 30 and for pileup 140 and 200. The modes of the three distributions are $0.3,1.2$, and $1.9 \mathrm{~mm}^{-1}$ and their means are $0.2,0.9$, and $1.4 \mathrm{~mm}^{-1}$, respectively. 


\section{The CMS MIP Timing Detector}

\subsection{Physics performance of CMS with the MTD}

The MTD will enhance the physics performance of CMS in two ways. One main application is the mitigation of pile-up effects on the physics object reconsruction. By tagging out of time tracks the reconstruction will be more precise, less biased and more efficient. In Figure 2 (left) we show the impact of this on the b-tagging efficiency. There is a significant improvement in the b-jet identification efficiency at an reduced mistag probability. This particular performance parameter has large impact on the yield of di-Higgs decays into two b-quarks and two photons, one of the primary physics goals of HL-LHC. The total yield is increased by $22 \%$, equivalent to approximately two years of HL-LHC operation.
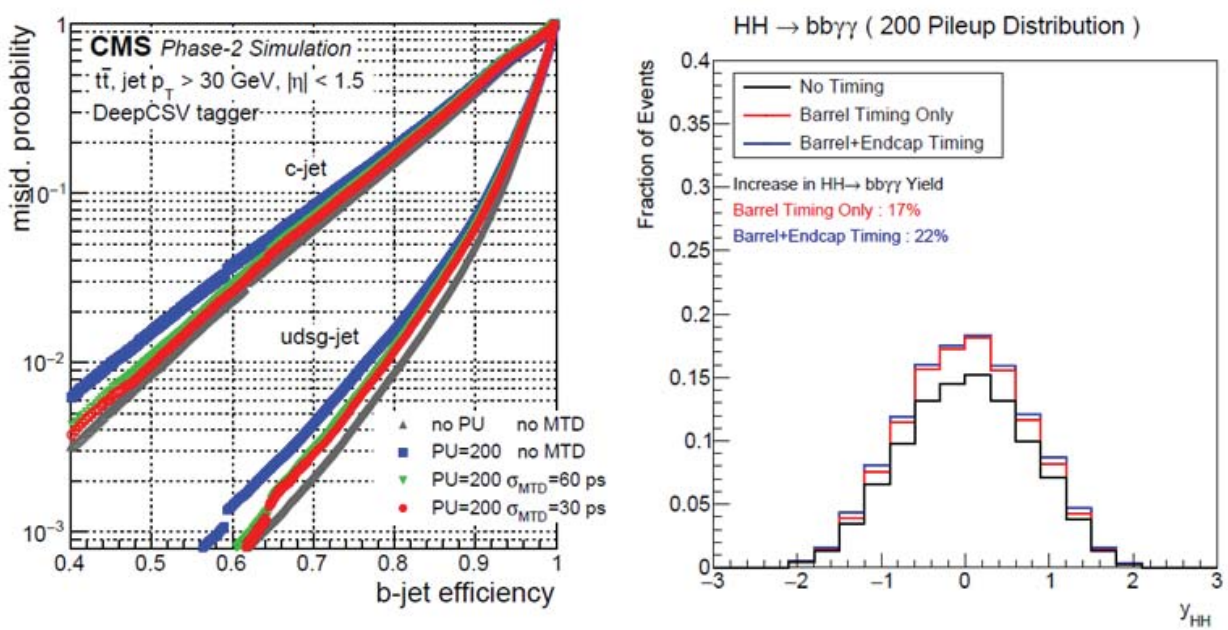

Figure 2: Improvement of the CMS physics performance with the inclusion of the MTD in the event reconsruction. (left) Secondary vertex tagging ROC curves for light and charm jets for $|\eta|<1$.5. Results with without (blue) and with timing for 30 (red) and 60 ps (green) resolution hypotheses are compared to the zero pileup case (grey). (right) Projections for the yield enhancement in $H H \rightarrow b b \gamma \gamma$ decays as a function of the Higgs boson rapidity. The distributions are normalized to the no-timing case.

A second benefit of the MTD is new physics analysis being enabled with the availability of precise time tagging of tracks. The ability to perform particle identification for pions and protons against kaons will give access to a new kinematic range in Heavy Ion physics as shown in Figure 3 (left). The 4D vertex reconstruction will allow to reconstruct the mass of long lived particle candidates, again providing access to a new kinematic range.

There are several other use cases which are only partially explored. It is expected to further expand the range of applications of the precision timing information provided by the MTD. Further details on the impact of the MTD on the CMS physics performance can be found in the TDR [3]. 

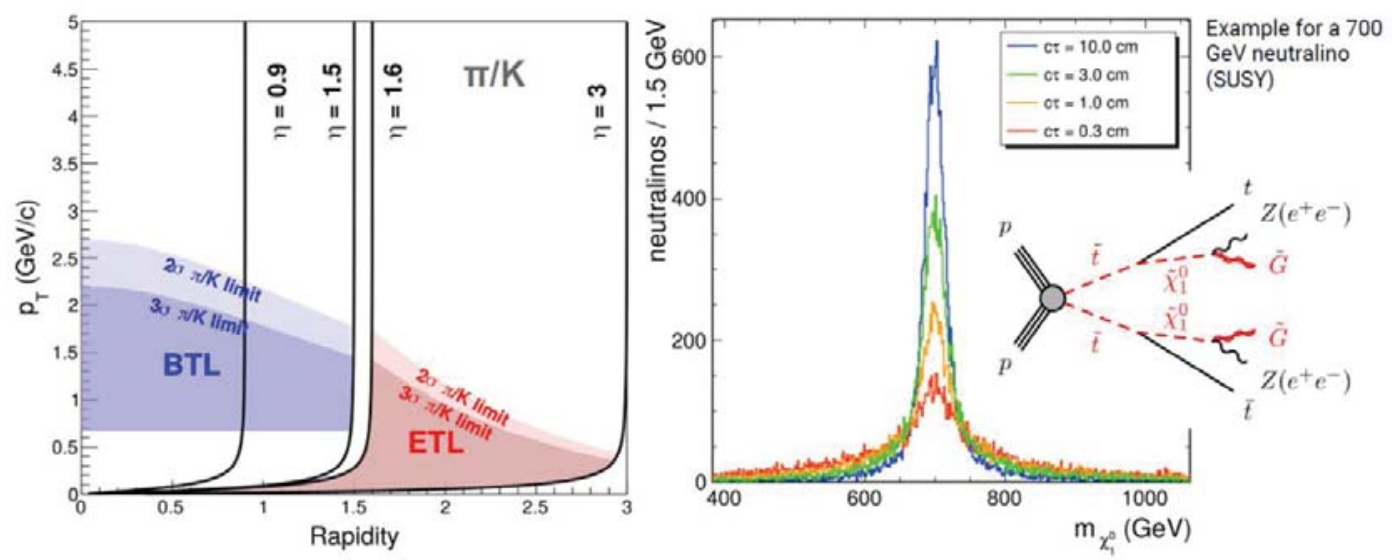

Figure 3 : (left) The MTD enabls measurements of the particle velocity for low pT hadrons. Shown is the particle ID capabilities which yield significant $\pi / K$ separation up to $2 \mathrm{GeV}$ and p/K separation up to $5 \mathrm{GeV}$. (right) The $4 \mathrm{D}$ vertex reconstruction of primary and secondary vertices will provides a closed kinematic for Long Lived Particles (LLP)and will allow to reconstruct LLP masses.

\subsection{Technology choices and detector layout of the MTDD}

The barrel part of the MTD uses LYSO scintilating crystals with SiPM readout as sensor technology. This technology provides the necessary radiation hardness and timing performance [4]. Further this technology is in wide use in industry, making it efficient and economic in construction and integration. The detector is segmented into approximately 331k channesl, each channel consisting of a LYSO crystal with a size of 3x3x57 mm3, read out by 2 SiPMs with about $3 \mathrm{~mm} 2$ active area.

The endcap part of the MTD uses Low Gain Avalance Diodes (LGAD) as sensors [5]. These diodes contain a highly doped $\mathrm{p}^{+}$region just below the $\mathrm{n}$ - implants increasing the electric field and providing an excellent time resolution while having an excellent radiation tolerance for the large fluences in the endcap $\left(<2 \times 10^{15} \mathrm{neq} / \mathrm{cm} 2\right)$. The size of the pixels is $3 \times 3 \mathrm{~mm} 2$ to keep the capacitance low. In order to optimize the numbers of sensors per production wafer the pixel are organized in small sensors of $21 \mathrm{x} 42 \mathrm{~mm}^{2}$. The time resolution and hit efficiency of the pixels have been studied in test beams showing an overall efficiency greater than 99\%.

The BTL will be mechanically attached to a carbon fiber tube, the tracker support tube (TST) which serves as the support structure and thermal and environmental enclosure of the CMS central tracker. The MTD will share services and controls with the CMS central tracker detector as well. The BTL is subdivided into 72 segments, referred to as trays, supported by rails from the inner wall of the TST.

The ETL will be housed in a dedicated thermal enclosure and mechanically attached to the front face of the CMS High Granularity Calorimeter (HGCAL). The ETL will feature two layers of LGAD sensors on each detector endcap side. Each disk is subdivided into four wedges for easier handling and assembly. Further details of the detector design of the MTD can be found in the technical design report. 


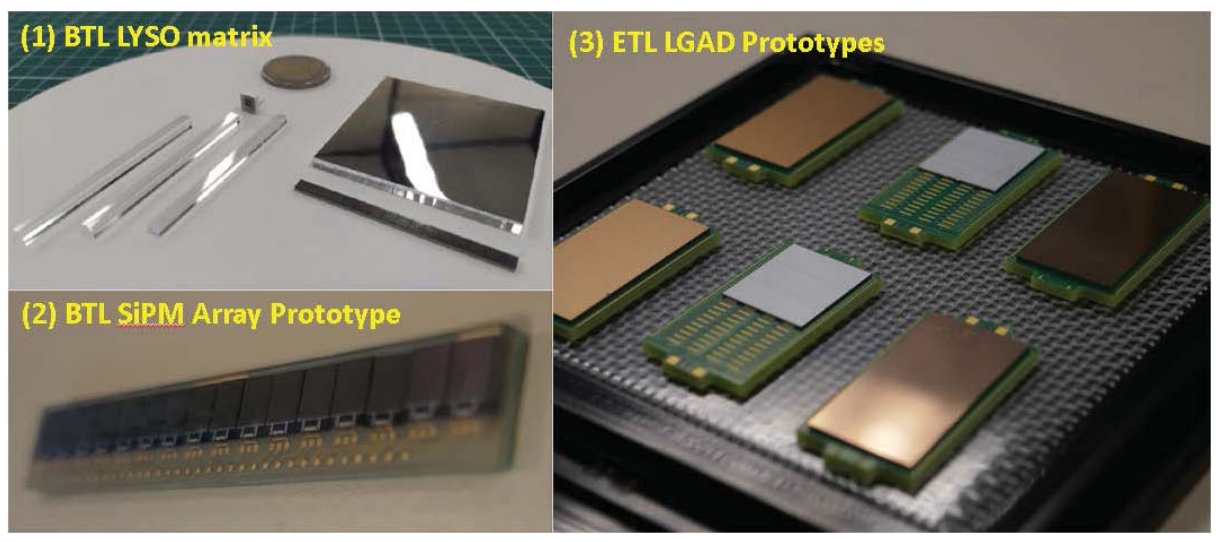

Figure 4: (left) LYSO matrix (1) and SiPM arrays (2) for the BTL. One SiPM arrays is glued on the end of the crystal matrix The LYSO matrix has a size of about $57 \times 57 \mathrm{~mm}^{2}$ with a thickness of $3 \mathrm{~mm}$. There will be a total of about 10000 such sensors in BTL. (right) ETL LGAD prototypes sensors (3), bump-bonded on PCBs. In the final design the sensors will have a size of about $21 \times 42 \mathrm{~mm}^{2}$ and there will be a total of about 16000 of such sensors in ETL.

\subsection{Detector Performance}

The detector performance of the MTD design has been validated in extensive test beams. In Figure 5 (left) we show the timing resolution of a single BTL channel. The timing information from a single channel combines the measurement from the two SiPMs at the end of the LYSO bar. The combined resolution show is around 30 ps along the lenght of the LYSO bar. In-situ, the performance is driven by the radiation induced dark count rate in the SiPMs which will increase the noise in the detector thoughout its lifetime. By optimizing the SiPM technology and by operating the detector at $-30 \mathrm{C}^{\circ}$, the resolution will remain below around 60 ps up to $3 \mathrm{ab}^{-1}$, the expected total integrated luminosity HL-LHC will accumulate. The ETL sensors are less sensitive to radiation. The main challenges are the optiization of the ultimate sensor timing performance which is limited by landau fluctuations to about 30 ps. This is compensated in ETL by implementing two layers of LGAD sensor on each endcap.
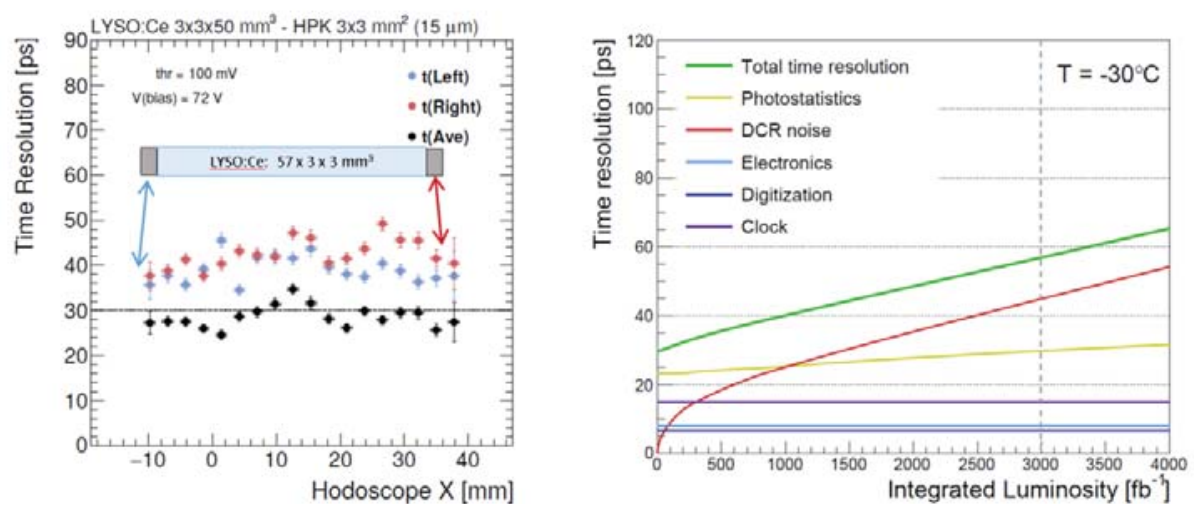

Figure 5: BTL sensor performance. (Left)Test beam results from a BTL sensor. Shown is the timeresolution as a function of the particle impact point along the LYSO bar. The performance for both of the SiPMs on the ends of the bar is shown as well as the combined performance. (right) BTL performance per channel as a function of the integrated luinosity the detector has recieved. The performance will degrade due to an increas in the dark count rate in the SiPMs. After an integrated luministy of $3 a b^{-1}$ the tme resolution will be around $55 \mathrm{ps}$. 
Further optimization is geared towards minimizing gaps between the sensor pixels to maximize the efficiency. The current generation of sensors has efficiencies above 99\% across the sensor area. In Figure 6 (left) we show the sensor efficiency aross 16 pixels. Figure 6 (right) shows the intrinsic time resolution and the jitter contribution, induced by landau fluctuations in the signal generation process in the silicon, to the total time resolution of about $30 \mathrm{ps}$. The double layer desing of the ETL will have two such measurements per track.
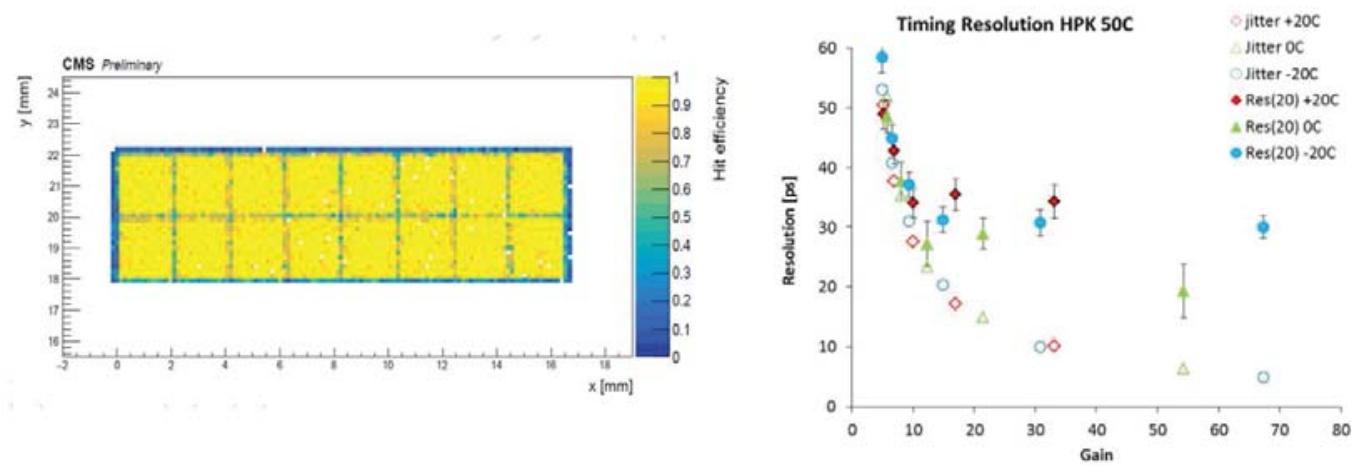

Figure 6(left) Efficiency of a 16-pad FBK sensor measured in an FNAL beam test with $120 \mathrm{GeV}$ protons. (right) Variation of the jitter and total time resolution as a function of the UFSD gain, as measured on a Hamamatsu $50 \mathrm{~mm}$ thick UFSD sensor. The jitter term decreases with gain while the total time resolution flattens around st $=30 \mathrm{ps}$.

\section{Summary}

The CMS Phase-2 upgrade program includes a MIP Timing Detector providing a 30-40 ps time resolution. This detector will be composed of two parts: the Barrel Timing Layer based on LYSO crystals and the Endcap Timing Layer based on silicon sensors (LGADs). The inclusion of timing information is expected to have a strong impact in the mitigation of the harsh pileup conditions at the HL-LHC, improving the physics object performance for jet reconstruction, btagging algorithms, lepton isolation, transverse missing momentum resolution and other observables. These improvements will translate into a sensitivity increase for important analysis such as the double Higgs search, and will also bring unique physics potential for complicated topologies such as those involving the production of long-lived particles.

\section{References}

[1] Apollinari, G. and Bruning, O. and Nakamoto, T. and Rossi, Lucio. High Luminosity Large Hadron Collider HL-LHC. CERN Yellow Rep. 5 1-19, 2015. 10.5170/CERN-2015-005.1

[2] Chatrchyan, S. and others. The CMS experiment at the CERN LHC. JINST, 3 S08004, 2008, 10.1088/1748-0221/3/08/S08004

[3] CMS Collaboration, A MIP Timing Detector for the CMS Phase-2 Upgrade, CERN-LHCC-2019003 ; CMS-TDR-020

[4] D. Anderson et al. On timing properties of LYSO-based calorimeters. Nucl. Instrum. Meth. A 794 (2015) 7, doi:10.1016/j.nima.2015.04.013.

[5] G. Pellegrini et al. Technology developments and first measurements of Low Gain Avalanche Detectors (LGAD) for high energy physics applications. Nucl. Instrum. Meth. A 765 (2014) 12, doi:10.1016/j.nima.2014.06.008 